\title{
Asymmetric impacts of near-Earth asteroids on the Moon
}

\author{
T. Ito $^{1}$ and R. Malhotra ${ }^{2}$ \\ 1 National Astronomical Observatory, Osawa 2-21-1, Mitaka, Tokyo 181-8588, Japan \\ e-mail: ito.t@nao.ac.jp \\ 2 Lunar \& Planetary Laboratory, University of Arizona, 1629 E. University Blvd., Tucson, AZ 85721-0092, USA
}

Received 14 July 2009 / Accepted 24 May 2010

\begin{abstract}
Context. Recent lunar crater studies have revealed an asymmetric distribution of rayed craters on the lunar surface. The asymmetry is related to the synchronous rotation of the Moon: there is a higher density of rayed craters on the leading hemisphere compared with the trailing hemisphere. Rayed craters represent generally the youngest impacts.

Aims. The purpose of this paper is to test the hypotheses that (i) the population of Near-Earth asteroids (NEAs) is the source of the impactors that have made the rayed craters; and (ii) that impacts by this projectile population account quantitatively for the observed asymmetry.

Methods. We carried out numerical simulations of the orbital evolution of a large number of test particles representing NEAs in order to determine directly their impact flux on the Moon. The simulations were done in two stages. In the first stage we obtained encounter statistics of NEAs on the Earth's activity sphere. In the second stage we calculated the direct impact flux of the encountering particles on the surface of the Moon; the latter calculations were confined within the activity sphere of the Earth. A steady-state synthetic population of NEAs was generated from a debiased orbital distribution of the known NEAs.

Results. We find that the near-Earth asteroids do have an asymmetry in their impact flux on the Moon: apex-to-antapex ratio of $1.32 \pm 0.01$. However, the observed rayed crater distribution's asymmetry is significantly more pronounced: apex-to-antapex ratio of $1.65 \pm 0.16$. Our results suggest the existence of an undetected population of slower (low impact velocity) projectiles, such as a population of objects nearly coorbiting with Earth; more observational studies of young lunar craters is needed to secure this conclusion.
\end{abstract}

Key words. minor planets, asteroids: general - Moon - planets and satellites: surfaces - methods: numerical

\section{Introduction}

It is well known that many satellites of the solar system planets are locked in synchronous rotation - their mean rotational angular speed and mean orbital motion is in a 1:1 commensurability. The synchronous rotation of these satellites leads to asymmetric spatial distribution of impact craters on these satellites: the leading hemisphere tends to have more craters than the trailing hemisphere. Such leading/trailing asymmetries in crater distributions have been observed on the Galilean satellites of Jupiter and on Neptune's moon Triton (e.g. Shoemaker et al. 1982; Schenk \& Sobieszczyk 1999; Zahnle et al. 1998).

Such an asymmetry was recently confirmed on the Moon. A detailed analysis of the Clementine 750-nm mosaic images has revealed that there is spatial variation in the density of rayed craters on the Moon (Morota \& Furumoto 2003). Lunar rayed craters are morphologically young and fresh craters with bright rays, generally estimated to be younger than 0.8 billion years old (e.g. McEwen et al. 1997). A total of 222 rayed craters larger than $5 \mathrm{~km}$ in diameter $(D)$ are identified in the study area of about $1.4 \times 10^{7} \mathrm{~km}^{2}$. The average density of rayed craters on the leading side of the Moon is found to be substantially higher than that on the trailing side, and the observed ratio of crater density $(D>5 \mathrm{~km})$ at the apex to that at the antapex is about 1.65. Based on a rough analytical estimate, Morota \& Furumoto (2003) conclude that this ratio suggests that recent craters on the Moon are formed mainly by the near-Earth asteroids which have lower impact velocities, rather than comets that have systematically higher impact velocities. Whether or not these conclusions are correct, it is true that the ratio of crater densities of the leading side and the trailing side of the Moon contains a significant amount of information about the type of projectile populations that have created craters on the lunar surface over the past $\sim 1$ billion years and under what kind of dynamical conditions.

The origin of the leading/trailing asymmetry of impact craters on a synchronously rotating planetary satellite is qualitatively explained as follows. Assume that the source of impacts is a heliocentric population of small objects on modestly eccentric and inclined orbits. In the rest frame of the planet, this population appears almost isotropic and the impact velocity vectors have an isotropic distribution. The impact craters asymmetry occurs because the satellite in synchronous rotation encounters projectiles more often on its leading side than on its trailing side. Furthermore, the average impact velocity of projectiles tends to be larger on the leading side than on the trailing side due to the difference of average relative velocities between the projectiles that encounter the leading and the trailing sides; this leads to systematically larger craters on the leading hemisphere compared with the trailing hemisphere.

The degree of the asymmetric crater distribution is a function of satellite's orbital velocity and the average relative velocity between projectiles and the planet-satellite system. When a satellite with synchronous rotation has a large orbital velocity around its mother planet, or when the average relative velocity between projectiles and the planet-satellite system is small, the asymmetric distribution of craters becomes more pronounced. Smaller orbital velocity of the satellite, or larger average relative 
velocity of projectiles tends to diminish the asymmetry of crater distribution.

The purpose of the present paper is to quantitatively test the hypothesis that impacts from the NEA population (with its currently known properties) account for the observed asymmetric crater distribution on the Moon. We do this by carrying out extensive numerical integrations of test particles to simulate the impact flux of NEAs. In order to obtain impact statistics and impact velocity distribution, we calculate direct impacts of projectiles on the Moon without analytical approximation. In Sect. 2 we describe our dynamical model, our numerical method and our choice of initial conditions, and a description of the first stage of our numerical simulations in which we trace the dynamical evolution of test particles from their initial locations to the edge of Earth's activity sphere; our results on NEA encounters with the Earth's activity sphere are given in Sect. 2.1. Section 3 describes the second stage of our numerical simulation in which we trace the evolution of particles within the Earth's activity sphere to obtain impact fluxes, impact velocities and their spatial distribution on the Moon. Section 4 provides a comparison of our simulation results with the observations of the lunar crater record. Section 5 is devoted to discussion of the results, and Sect. 6 to a short summary and conclusions.

While our work was in progress, Gallant et al. (2009) published a study with quite a similar motivation to ours, which also yielded a similar numerical result about the expected lunar cratering asymmetry from NEA impacts. Although a large part of our results overlap, the numerical models are different. As we describe in Sect. 2, our numerical model is straightforward and includes the orbit evolution of NEA-like particles from their current orbits up to their impacts, while Gallant et al. (2009)'s study uses the NEA orbits without dynamical evolution. In this regard our paper serves as a complement to Gallant et al. (2009). We also consider some additional important implications of the results that were not discussed previously.

\section{Numerical model}

Our numerical model comprises of two stages. In the first stage, our numerical integrations include the eight major planets and the Sun, and a large steady-state number of test particles with NEA-like orbits. We numerically integrate their orbital evolution for up to 100 million years. Throughout these integrations, we record all close encounters of the particles that reach the Earth's activity sphere. (Note that in the first stage of calculation the Moon is not included.) We use this record in our second stage of numerical simulation, in which we adopt the restricted $N$-body model consisting of the Earth, the Moon, and the Sun, and cloned test particles within the Earth's activity sphere (as described in detail in Sect. 3). In the second stage, we do not include the effects of any planets save the Earth but we include the Moon's gravity.

For our first stage numerical simulation we generated a synthetic population of particles with orbital elements similar to the "debiased" distributions of near-Earth objects (NEOs) described in Bottke et al. (2002). (Note that NEOs are largely composed of NEAs, so we will keep using the term NEAs rather than NEOs in this paper.) Specifically, we generated 18000 particle initial conditions whose distributions of $a, e$, and $I$ obey the histograms shown in Fig. 12 of Bottke et al. (2002) which gives the debiased orbital distribution of the near-Earth asteroids of absolute
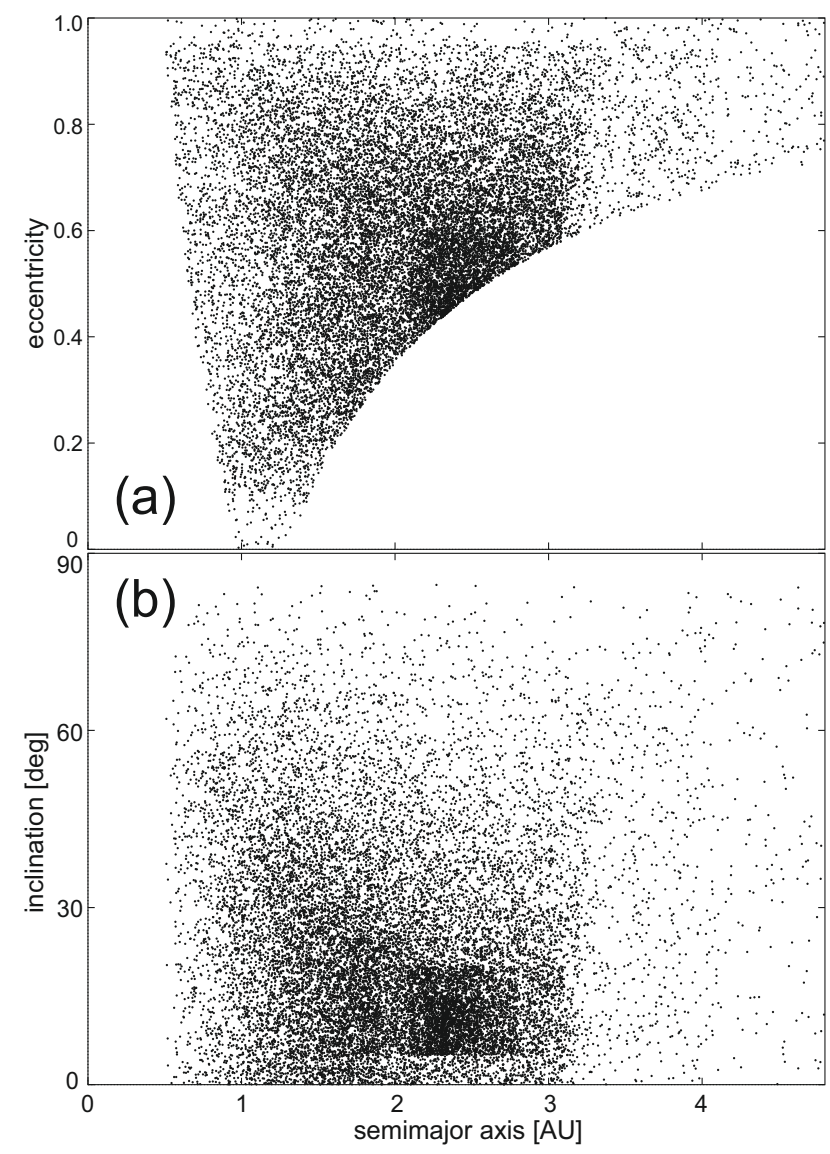

Fig. 1. Initial osculating orbital elements of the NEA-like particles in our numerical model. a) Semimajor axis vs. eccentricity. b) Semimajor axis vs. orbital inclination. (Inclinations are referred to the ecliptic at J2000.0.)

magnitude $H<18^{1}$. The orbital elements of our synthetic NEA population are shown in Fig. 1. This population represents a good snapshot of current orbital distribution of NEAs. Studies of impact craters in the inner solar system indicate that there has been a relatively constant supply of impactors over the past three billion years which has kept the impactor flux around the Earth-Moon system close to a stationary state (McEwen et al. 1997; Ivanov et al. 2002), and that this impactor population is dominated by NEAs (Strom et al. 2005).

For the numerical integration scheme we used the regularized mixed-variable symplectic method (Levison \& Duncan 1994). The basic framework of our first stage simulation follows Ito \& Malhotra (2006). When a test particle approaches within the physical radius of the Sun or that of planets, we consider the particle to have collided with that body and lost from the NEA population. Also, when the heliocentric distance of a test particle exceeds $100 \mathrm{AU}$, the particle is considered lost. Over the 100 Myr length of the simulation, a large fraction ( $90 \%$, e.g. Ito $\&$ Malhotra 2006) of the synthetic population would be expected to be removed in this way, and if this loss were not compensated, we would not be able to mimic a steady-state NEA flux. We realize the steady-state NEA flux in our numerical simulation as follows: for each "lost" particle, we immediately introduce in our simulation another particle with the original position and velocity of that "lost" particle. This procedure achieves a steady-state

\footnotetext{
${ }^{1}$ Note that at this stage of our calculation we do not consider at all the size-frequency distribution (or absolute magnitude distribution) of the particles.
} 
population of NEAs in our simulation. In particular, we verified that the distribution of lunar impact velocity remains steady throughout the simulation timespan.

\subsection{Particle encounters with Earth's activity sphere}

Over the 100 Myr simulation of a steady-state swarm of 18000 particles, we found 3998 collisions with Earth. We note that, although the number of planetary collisions is not large in our first stage numerical integrations, there are many more encounters at the planetary activity sphere of Earth. The activity sphere, also known as the sphere of influence, has a radius of $(m / M)^{2 / 5} d$ where $m$ is the mass of a planet, $M$ is the mass of the Sun, and $d$ is the heliocentric distance of the planet (Danby 1992). Earth's activity sphere, hereafter denoted $r_{\mathrm{I}}$, is about 144 times as large as the Earth's radius. In our first stage numerical simulation, we recorded the encounters of particles at the Earth's activity sphere over the 100 Myr integration, and found 42099969 encounters. This number is large enough to establish a time-dependent orbital distribution function of the particles, $F(a, e, I, \omega, \Omega, l ; t)$ that can be used to create "clones" of particles in order to increase the reliability of the collision statistics between the particles and the Earth or the Moon, as we describe in the next section.

In Fig. 2 we show time-integrated distribution (over the 100 Myr duration of our simulation) of encounter velocity components and encounter density (number of encounters per unit surface area) at the Earth's activity sphere of the particles. In our simulation the average encounter velocity of the particles at the Earth's activity sphere is $22.5 \mathrm{~km} \mathrm{~s}^{-1}$. We show these distributions along all three spatial directions, $x, y$ and $z$. There are several noteworthy features in these distributions. While the encounter velocity distributions with respect to the $x$ and $z$ directions are symmetric, the $y$-direction distributions are markedly asymmetric. Because of its very small orbital eccentricity, the Earth's orbital motion is practically along the $+y$ direction. We see the expected effect that more particles encounter the Earth's activity sphere on its leading side (from the positive $y$ direction) than its trailing side: the fraction of encountered particles having negative $v_{y}$ is larger than that of the particles having positive $v_{y}$ (the panel (a)). Consistent with this, we see in the panel (b) that the encounter density is higher on the leading hemisphere (0-90 angle with respect to the leading point in the $y$ direction), and lower on the trailing hemisphere.

In the panel (b), we also notice a pattern in the encounter density distributions along the $x$ direction: the encounter density of the particles is somewhat lower around the angle $\sim 90^{\circ}$ (with respect to the leading point of the $x$ direction, $\left(r_{\mathrm{I}}, 0,0\right)$ ). The pattern of distribution in the $x$-direction is understood by the following consideration. Because of its very small orbital eccentricity, the Earth's orbital motion is practically along the $+y$ direction. Along the $y$ axis, the angle with respect to the $x$ - leading point, $\left(r_{\mathrm{I}}, 0,0\right)$, is $90^{\circ}$; this defines the solar terminator at the Earth's activity sphere. Particles that encounter Earth in this region have very small velocity relative to Earth, particularly when their random orbital velocity is low; the lower average encounter velocity leads to the lower encounter frequency of particles in this region. This accounts for the dip near angle $90^{\circ}$ for the $x$ curves in the panel (b). Moreover, this effect would get smaller when the particle population has larger random orbital velocity.

We also notice a pattern in the encounter density distributions along the $z$ direction in Fig. 2b. However, the dips near $90^{\circ}$ are of noticeably smaller magnitude in this direction than in the $x$ direction, and the sign is the opposite: we see a concentration
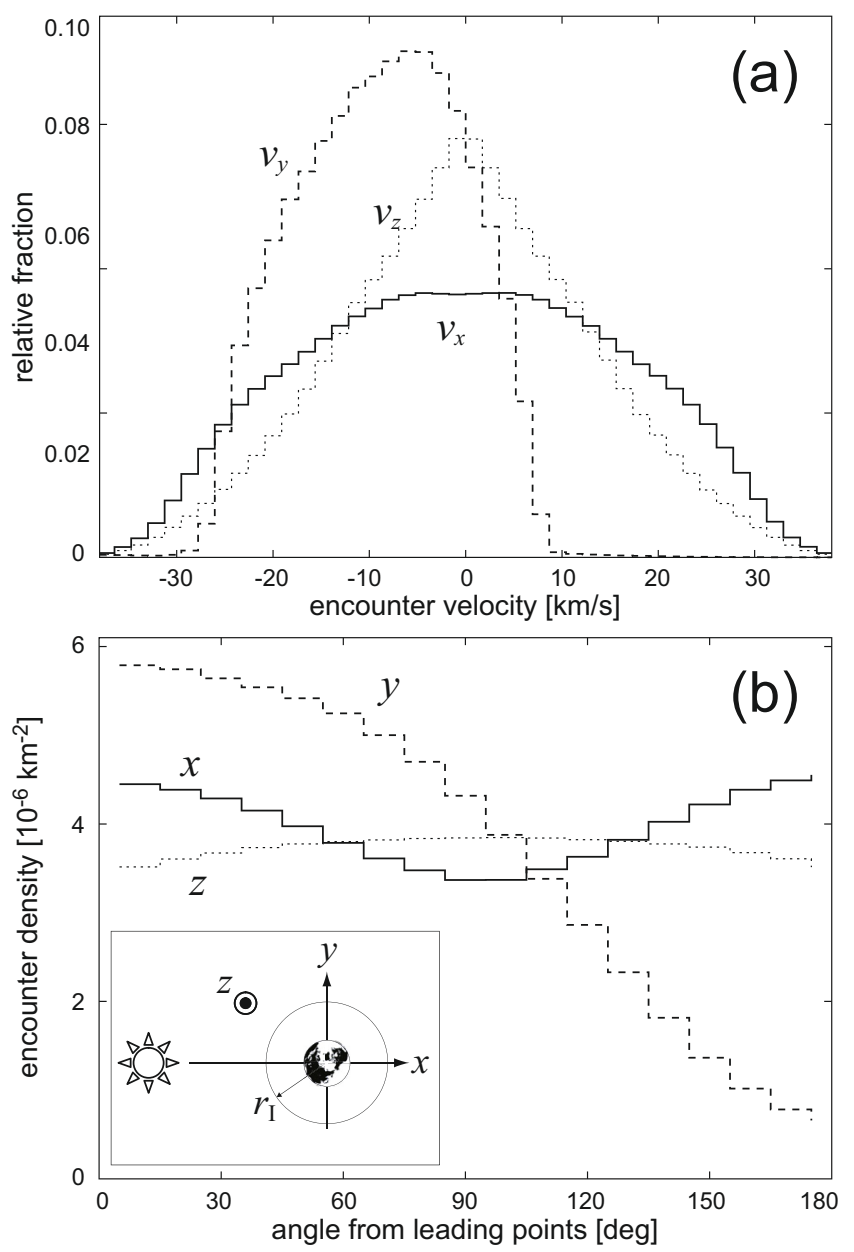

Fig. 2. Statistics of NEA-like particle encounters at the Earth's activity sphere, $r_{\mathrm{I}}$. a) Distribution of encounter velocity components $\left(v_{x}, v_{y}, v_{z}\right)$; the horizontal unit is $\mathrm{km} \mathrm{s}^{-1}$. b) Surface density of encounters at the surface of the Earth's activity sphere as a function of the angle from the leading points of each of the $x, y$, and $z$ directions. The vertical unit is $10^{-6} \mathrm{~km}^{-2}$. The inset in the panel b schematically shows the coordinate system $(x, y, z)$ adopted for this figure: the Sun always lies along the $-x$ direction, $+z$ is the normal to the Earth's orbit, the Earth practically goes toward $+y$ direction, and $r_{\mathrm{I}}$ is the radius of Earth's activity sphere. Coordinate of the leading point for the $y$ data in the panel is $\left(0, r_{\mathrm{I}}, 0\right)$.

of encounters around $90^{\circ}$. We note that the vertical scale height of the NEA-like particle population, approximately given by the average $\langle a \tan I\rangle$, is much larger than the radius of the Earth's activity sphere $\left(r_{\mathrm{I}} \sim 0.006 \mathrm{AU}\right)$. This is why the encounter frequency of the particles does not vary as much along the $z$ direction compared with the $x$ direction.

From the above results on the distribution of particle encounters on the Earth's activity sphere shown in Fig. 2, it is clear that the Earth receives more NEA impacts on the leading (positive $y$ ) hemisphere than on the trailing hemisphere. This asymmetry leads to AM/PM asymmetries in NEA impact events, which is discussed in Gallant et al. (2009) in detail.

\section{Asymmetric impacts on the Moon}

Using the particle encounters at Earth's activity sphere, we generated cloned particles by perturbing the encounter position $r$ and velocity $\boldsymbol{v}$ of each of the original particles so that their initial trajectories at the activity sphere become slightly different: $\boldsymbol{r}_{\text {clone }}=\left(1+\delta_{r}\right) \boldsymbol{r}_{\text {original }}$ and $\boldsymbol{v}_{\text {clone }}=\left(1+\delta_{v}\right) \boldsymbol{v}_{\text {original }}$, where $\delta_{r}$ 
and $\delta_{v}$ are random numbers in the range $[-0.1,0.1]$. This procedure produces a large number of particles that obey nearly the same orbital distribution function as the original particles (" $F$ " in the previous description, see Fig. 2) but with somewhat different paths toward the Earth (and the Moon).

We repeated this cloning procedure five hundred times from the result of the first stage numerical integrations, generating 21.049895 billion particle initial conditions on the Earth's activity sphere. Using these sets of cloned particles, we performed a second set of numerical integrations, this time with the restricted $\mathrm{N}$-body problem including the Sun, the Earth, the Moon, and the cloned test particles. Here we did not include the effect of other planets than the Earth, but we included the Moon's gravity. All the cloned particles started near the Earth's activity sphere, and were integrated until they hit the Earth or the Moon or went out of the sphere. We used the present orbital elements of the Moon with true anomaly randomly chosen from 0 to $360^{\circ}$ for each of the 500 sets of clones. We employed the regularized mixedvariable symplectic method again with a stepsize of $84.375 \mathrm{~s}$ $\left(=2^{-10}\right.$ days). (This small step size was arrived at by a process of trial to ensure that even high velocity particle collisions with the Moon were not missed.) Calculations were carried out in the geocentric frame.

The second stage numerical integrations yielded 1509364 collisions with the Earth and 73923 collisions with the Moon. Figure 3 shows the distribution of impact velocities and impact angles on the Earth and on the Moon. Overall, the average impact velocities of the clones on the lunar surface $\left(\sim 22.4 \mathrm{~km} \mathrm{~s}^{-1}\right)$ is almost the same as the average encounter velocity of the original particles at the Earth's activity sphere. This means that lunar gravity plays only a minor role in accelerating particles to the lunar surface in our numerical model. Not only lunar gravity but the Earth's gravity also plays only a small role: average impact velocity of the clones at the Earth's surface is $\sim 23.1 \mathrm{~km} \mathrm{~s}^{-1}$, not being very different from the average impact velocity with the lunar surface, in spite of the large difference of the escape velocities from the two bodies $\left(\sim 11.2 \mathrm{~km} \mathrm{~s}^{-1}\right.$ on the Earth and $\sim 2.4 \mathrm{~km} \mathrm{~s}^{-1}$ on the Moon). The ratio of the number of collisions with the Earth and those with the Moon is found to be $20.4 \pm 0.1$. For comparison, we note that Zahnle \& Sleep (1997) reported the ratio of collisional cross sections of the Earth and the Moon as $\sim 23$, by assuming isotropic collisions and average impact velocity of Earth-crossing asteroids to be $16.1 \mathrm{~km} \mathrm{~s}^{-1}$ on the Earth.

Regarding the impact angles on the lunar surface, we note that from simple geometrical considerations for an isotropic distribution of impact direction, the impact angle distribution is expected to have a probability density function proportional to $\sin 2 i$ (Shoemaker 1962) where $i$ is impact angle and $i \rightarrow$ 0 means oblique impact. The results of our simulation, taken all together, are consistent with isotropic impact angles (Fig. 3b). We can mention in passing that there is a small but noticeable difference in the impact angle distribution on the leading and trailing hemispheres of the Moon: the trailing hemisphere slightly disfavors oblique impacts whereas oblique impacts are slightly enhanced on the leading hemisphere, Fig. 3b. This is not of significance for the statistical results in the present paper, but it may be of interest for future studies of individual lunar craters.

It is also interesting to examine the orbital element distribution of the lunar colliders. In Fig. 4, we plot histograms of the distribution of $a, e, I$ for the lunar colliders as well as for our synthetic NEA initial conditions; for the lunar colliders, we plot histograms of both their initial orbits and their orbits just before impact with the Moon. Comparison of the two initial orbit distributions shows that the lunar collider population has a higher
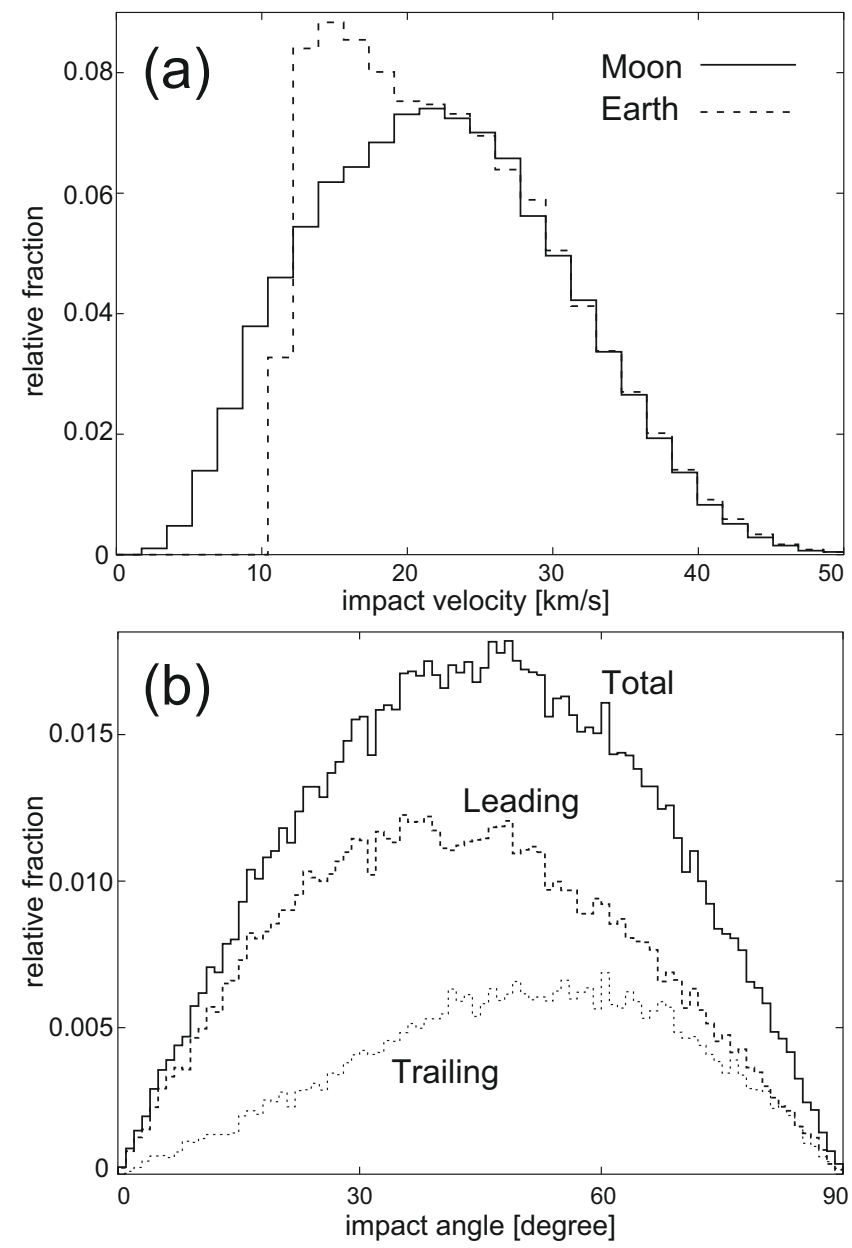

Fig. 3. Statistics of impactors on the Moon and on the Earth. a) Distribution of impact velocity on the Moon (solid line) and on the Earth (dashed line) of the clones. b) Distribution of impact angle on the Moon on the entire surface (solid line; denoted as "Total"), on the leading hemisphere (dashed line; denoted as "Leading"), and on the trailing hemisphere (dotted line; denoted as "Trailing").

fraction of low inclinations and low semimajor axes compared to the overall NEA initial orbit distribution. Comparison of the initial orbits and final (just before impact) orbits of the lunar colliders shows that there is significant dynamical evolution of orbital elements that occurs prior to lunar impact: on average, semimajor axes evolve to lower values, eccentricities and inclinations evolve to higher values. This evolution takes place during the first several million years of their trajectories.

\section{Simulation compared with lunar crater data}

The second stage of our numerical simulation yields the spatial distribution of NEA impacts on the lunar surface. As mentioned in the introductory section, in order to compare the distribution of impacts in our numerical model with the actual lunar crater record, we have to consider a correction to the raw numerical results due to the systematic difference in the impact velocities on the leading and trailing hemispheres, a difference that owes to the orbital motion of the satellite about its mother planet. This correction, which turns out to be quite small for the Moon, arises as follows.

For a satellite with synchronous rotation, the average impact velocity of projectiles is somewhat larger on the leading side than on the trailing side. This difference means that, on average, 
T. Ito and R. Malhotra: Asymmetric cratering on the Moon
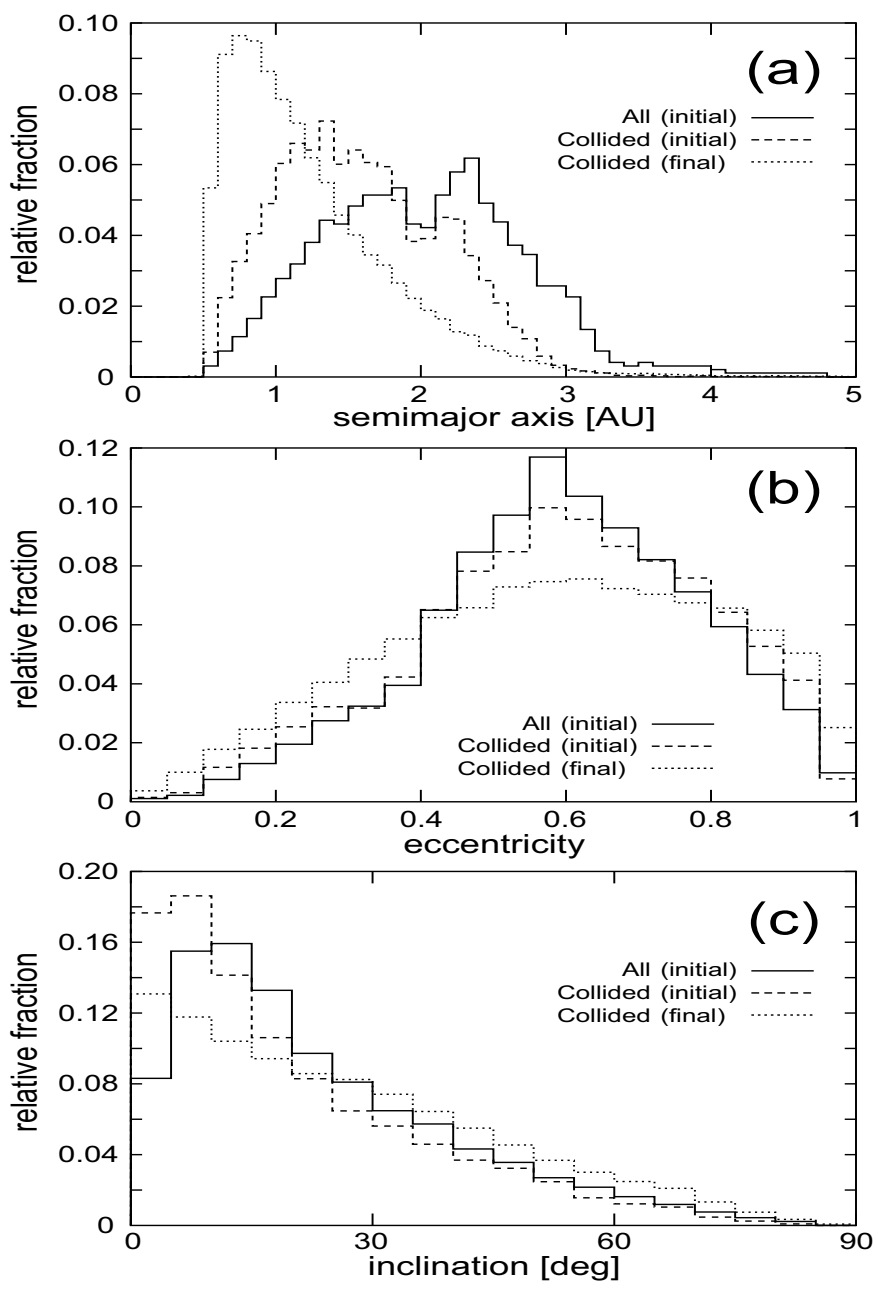

Fig. 4. Heliocentric orbital distribution of a) initial semimajor axis distribution of all particles included in the first stage integrations (solid line), initial semimajor axis distribution of the particles (their equivalent clones) that eventually collided with the Moon (dotted line), and final semimajor axis distribution of the particles (their equivalent clones) that eventually collided with the Moon (dashed line; i.e. when they hit the lunar surface). Note that the dip around $2 \mathrm{AU}$ in the solid line distribution is originated from the debiased semimajor axis distribution of Bottke et al. (2002). b) Same as a), but for eccentricity. c) Same as a) and $\mathbf{b})$, but for orbital inclination.

the apparent crater size would be larger on the leading side than on the trailing side (assuming the projectile size-frequency distribution (SFD) is not different on the two sides). To illustrate the effect this has on the crater densities, consider a power law SFD of craters, as in the solid line shown schematically in Fig. 5 where $N$ is the cumulative number of craters per unit area. As a result of the higher (lower) average impact velocity on the leading (trailing) side, the SFD curve of the craters on the leading (trailing) side becomes shifted toward the positive (negative) direction along the horizontal $(D)$ axis, as indicated by the arrow (1) in the figure. This horizontal shift is practically equivalent to a vertical shift of the SFD curve toward the dotted line in Fig. 5, as indicated by the arrow (2) in the figure, illustrating that $N$ gets larger on the leading side (smaller on the trailing side) for the entire range of crater diameter, $D$.

The magnitude of the shift depends upon the relationship between the impact velocity $v_{\text {imp }}$ and the crater size, $D$. Here we employ the Pi-group scaling (Schmidt \& Housen 1987; Melosh 1989; Housen et al. 1991) where approximately $D \propto v_{\text {imp }}^{\alpha}$

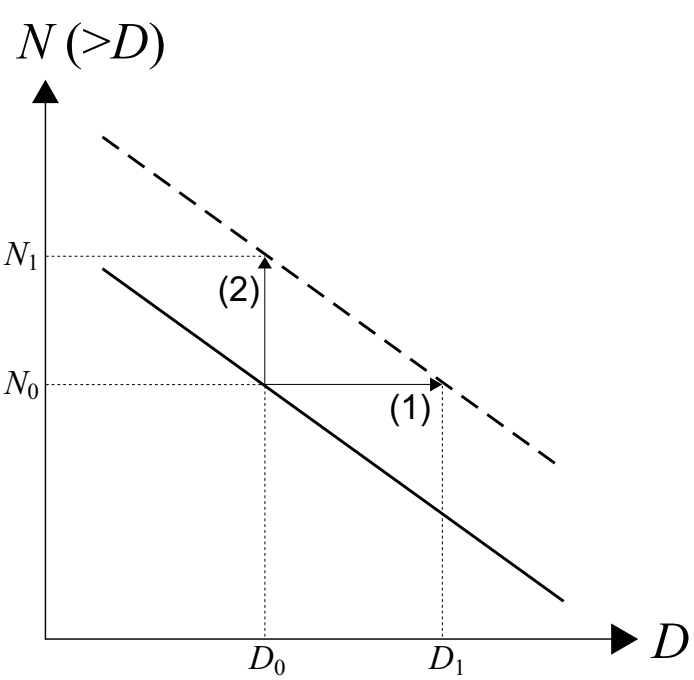

Fig. 5. Schematic illustration of the change of crater SFD due to apparent change of crater size. $D$ is crater diameter, and $N(>D)$ is the cumulative number of craters whose diameter is greater than $D$. Adopted and modified from Ishizaki \& Furumoto (1997).

with $\alpha=0.44$. For the cumulative SFD of craters, we adopt ${ }^{2}$ $N(>D) \propto D^{\beta}$ with $\beta=-2$ which represents well the SFD of the young rayed craters (Morota $\&$ Furumoto 2003). When the average impact velocity is changed from $v_{0}$ to $v_{1}$, the cumulative number density of craters at any given diameter $D$ changes from $N_{0}$ to $N_{1}=N_{0}\left(v_{1} / v_{0}\right)^{-\alpha \beta}$. This holds for any values of $D$ as long as the crater SFD is expressed by a single power law.

From the results of our second stage simulation, we computed the average impact velocity, $\left\langle v_{\text {imp }}\right\rangle$ in $\mathrm{km} \mathrm{s}^{-1}$, of NEAs on the lunar surface as a function of angle from apex, $\gamma$ (degrees), by a least squares fit. We find $\left\langle v_{\text {imp }}\right\rangle=-0.00672 \gamma+22.7$. This indicates that difference of $\left\langle v_{\text {imp }}\right\rangle$ between the $\gamma=90^{\circ}$ point and the apex $(\gamma=0)$ or antapex $\left(\gamma=180^{\circ}\right)$ is less than $0.61 \mathrm{~km} \mathrm{~s}^{-1}$. Compared with the average of $v_{\text {imp }}$ over the entire range of $0 \leq \gamma \leq 180^{\circ}$, these velocity differences amount to $\$ 2.71 \%$, thus corresponding corrections to the cumulative SFD are given by $N_{1} / N_{0} \sim 1.02$. This small difference is owed to the fact that the lunar orbital velocity of $\sim 1 \mathrm{~km} \mathrm{~s}^{-1}$ is much lower than the average impact velocity $\left\langle v_{\text {imp }}\right\rangle$ of $\sim 22 \mathrm{~km} \mathrm{~s}^{-1}$. As a result of this small dependence, apparent change of the crater SFD due to the impact velocity difference between the leading side and the trailing side is quite modest. This effect, however, would be important when considering the asymmetric crater distribution on a satellite with higher orbital velocity around its mother planet such as Ganymede around Jupiter (Shoemaker et al. 1982; Zahnle et al. 2001).

Including this correction to our second stage simulation, we computed the simulated spatial density of NEA impacts on the Moon. Normalizing to unity at antapex, our simulation result for the crater density as a function of apex angle are shown in Fig. 6, panel (a). In Fig. 6, we used a simple sinusoid with the function form of $A+B \cos \gamma$ for a fitting curve where $A$ and $B$ are fitting parameters, normalizing $A+B \cos 180^{\circ}=1$.

For comparison, panel (b) shows the distribution found from the analysis of observed lunar rayed craters (Morota \& Furumoto 2003). Note that the number of the lunar rayed craters in the observational data analyzed by Morota \& Furumoto (2003) is only

2 This SFD is also consistent with the young crater populations linked to impacts by the NEA population on all terrestrial planets and the Moon (Strom et al. 2005). 


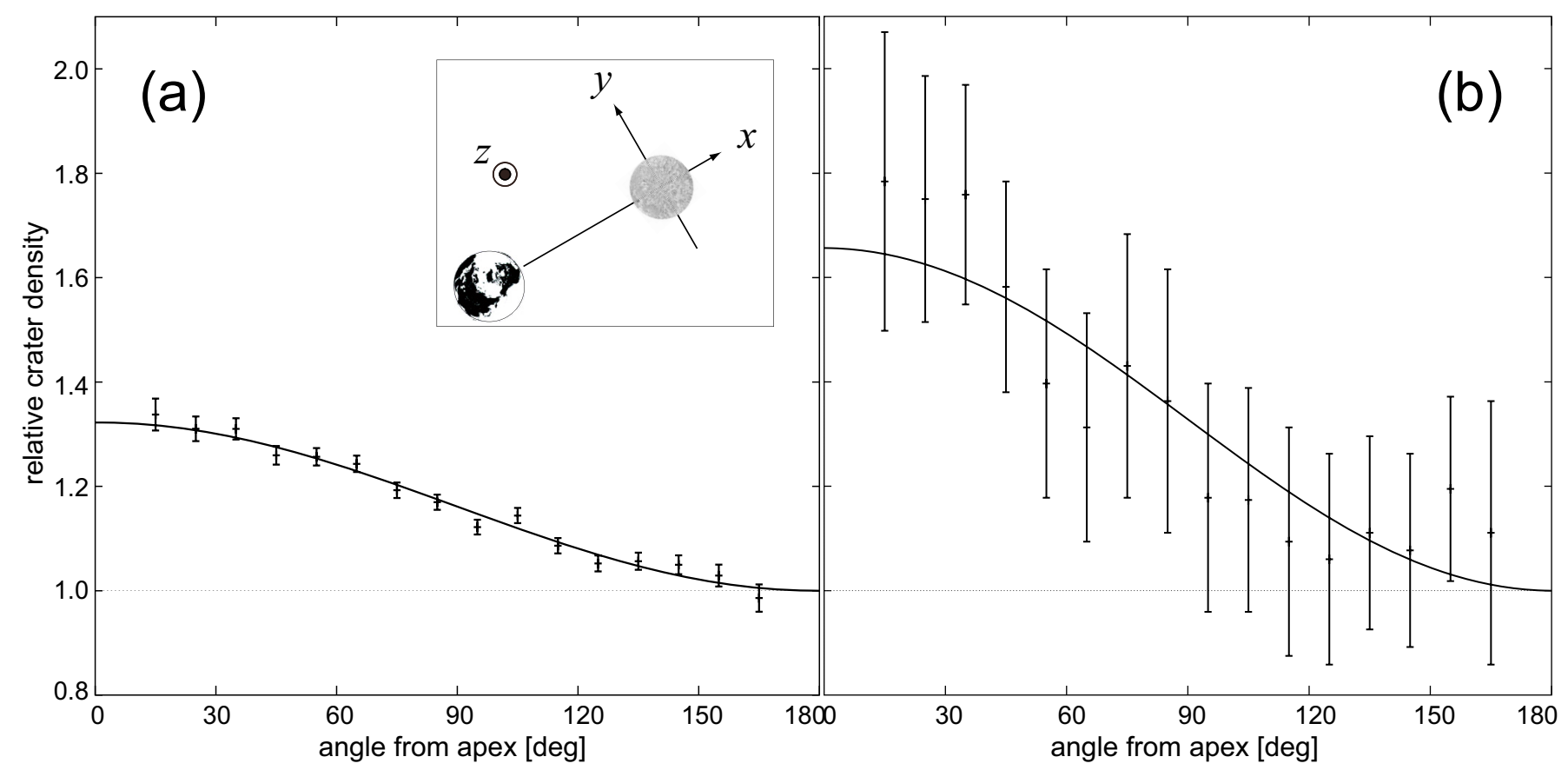

Fig. 6. Modeled and observed impact crater distribution on the Moon. We normalized the crater density to unity at antapex $\left(\gamma=180^{\circ}\right)$ using the best fit sinusoid (solid line curve). a) Numerical result including the correction due to the difference of average impact velocity as a function of the angular distance $(\gamma)$ from apex $(\gamma=0)$. b) The observed rayed crater distribution of $D>5 \mathrm{~km}$ (Morota \& Furumoto 2003). The inset in a) illustrates the coordinate system in this frame: the Earth always lies along $-x$ direction, the Moon velocity is toward $+y$ direction, and $+z$ is the north of the Earth-Moon system. Apex point is defined as $(x, y, z)=\left(0, R_{\mathrm{M}}, 0\right)$ where $R_{\mathrm{M}}$ is the lunar radius.

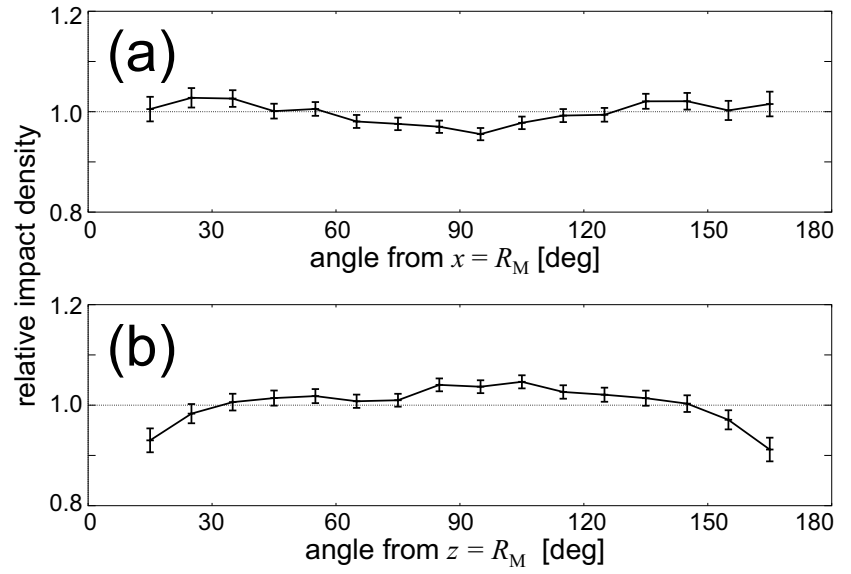

Fig. 7. Relative impact density (impact flux) on the Moon in our numerical results a) along $x$-axis with respect to the point $\left(R_{\mathrm{M}}, 0,0\right)$, and b) along $z$-axis with respect to the point $\left(0,0, R_{\mathrm{M}}\right)$. The average impact density over the range of angles 0 to $180^{\circ}$ (from $x=R_{\mathrm{M}}$ for the $x$ direction and from $z=R_{\mathrm{M}}$ for the $z$ direction) is normalized to unity. Note that the normalization here is different from that in Fig. 6.

222, while we have about 74000 impacts in our simulation. This difference is reflected in the difference of the errorbar magnitudes in Fig. 6, which are based on Poisson statistics.

Examining Fig. 6, what we notice first is that the apex/antapex asymmetry is less prominent in the numerical results (panel (a)) compared with the observed lunar rayed crater record (panel (b)). The maximum crater density at apex is about 1.65 (normalized to unity at antapex, and estimated from the best-fit sinusoid) in the observed crater record, whereas in our simulations, it is $1.32 \pm 0.01$.
We also examined our numerical model result for trends in the NEA impact density (impact flux) with respect to the angles along the $x$ and $z$ axes (Fig. 7). Here we again adopt Poisson statistics to assign uncertainties in the data plotted in Fig. 7. We find a tiny dip around the angle $90^{\circ}$ along the $x$ direction (Fig. 7a), although the numerical noise is large. We can interpret this dip as related to the dip that we see in the encounter statistics of particles at Earth's activity sphere along the $x$ axis (cf. Fig. 2b) and as owing to the same dynamical reason.

Along the $z$ axis, we notice a lower impact density at the polar regions (Fig. 7b). We interpret this pattern as related to the encounter density at the Earth's activity sphere along the $z$ direction, Fig. 2b. That the number of particle encounters at Earth's activity sphere becomes the smallest around the angle $=90^{\circ}$ is reflected in the trend found in Fig. 7b. The difference in cratering rate between at the polar and the equatorial regions is $\sim 10 \%$; this is consistent with the analytical estimate by Le Feuvre \& Wieczorek (2008), as well as the numerical result presented in Gallant et al. (2009).

\section{Discussion}

Does the dynamical model of NEA impacts account for the observed asymmetry of lunar rayed craters? The observed crater record has relatively large errorbars compared to our dynamical model results (Fig. 6), and the apex/antapex contrast of the observed and modeled crater densities may be considered at least qualitatively consistent with the observations. Such a conclusion would imply that the young lunar craters are owed to impacts of the NEAs whose orbital distribution we already know.

However, the intriguing systematic difference between the degree of asymmetry between our dynamical model and the observed crater record, though not enormous, is worthy of 
comment. If we use the best-fit sinusoids in Fig. 6, we can say that the dynamical model accounts for only about $49 \%$ $(=(1.32-1) /(1.65-1))$ of the observed lunar rayed crater asymmetry. We would like to pursue the reasons for this potential discrepancy, hoping to understand and constrain better the dynamical origin of the projectiles that have created the lunar rayed craters over the past $\sim$ one billion years. Because the uncertainties in our numerical model are much smaller than those in the observational data (compare the errorbars in Figs. 6a and b), we must be very cautious in the discussion of this point. We emphasize that the possible explanations discussed below must await assessment with improved observational data.

One possible explanation is related to the impact velocity distributions. The leading/trailing asymmetry becomes more prominent when the average relative velocity between the Moon and the projectiles is low. The NEA-like particles are, by their dynamical definition, the "slowest" (relative to Earth) among all the known small body populations in the solar system. That even these slow particles may not fully account for the observed asymmetric distribution in the lunar crater record suggests that there may exist a presently-unobserved population of small objects near the Earth's orbit that have even lower average relative velocity than the currently known near-Earth asteroids do. Conventional debiasing techniques, such as in Bottke et al. (2000, 2002), would not enhance the NEA-like particles with low relative velocity. Rather, such debiasing generally compensates for the existence of more distant objects with higher relative velocity (i.e., larger $e, I$ ).

This argument predicts the existence of a hitherto unseen population of slow objects whose heliocentric orbits are close to the Earth-Moon system. We make a rough estimate of the unseen population as follows. The best-fit sinusoid to the observational lunar crater asymmetry is consistent with an impactor population with average lunar impact velocity of $10-12 \mathrm{~km} \mathrm{~s}^{-1}$ (Morota \& Furumoto 2003). Consider an impactor population having a similar shape of the impact velocity distribution function as the simulated NEAs (Fig. 3a) but with $\left\langle v_{\text {imp }}\right\rangle=10-12 \mathrm{~km} \mathrm{~s}^{-1}$ rather than the $\sim 22 \mathrm{~km} \mathrm{~s}^{-1}$ that we found in our dynamical model based on the known NEAs. In such a population, the fraction of slow objects would be roughly $50 \%$ more than the fraction of slow objects in the currently known population of NEAs; here we define "slow" NEAs as those having potential lunar impact velocity $<12 \mathrm{~km} \mathrm{~s}^{-1}$; such objects would be nearly coorbiting with Earth. In other words, our rough estimate of the actual slow NEA population is $\sim 50 \%$ more than the known slow NEAs.

Such a population could have remained undetected in observational surveys to date either because the surveys have low sensitivity to their orbital parameters or because these objects are fainter (smaller and/or darker). The rayed crater record in Fig. $6 \mathrm{~b}$ contains craters with diameter $D>5 \mathrm{~km}$. On the lunar surface, a crater with $D \approx 5 \mathrm{~km}$ can be created by an asteroidal projectile with $D_{\text {projectile }}=0.2-0.3 \mathrm{~km}$ even when the impact velocity is as small as $10 \mathrm{~km} \mathrm{~s}^{-1}$ and when the projectile density is that of porous rock $\left(\sim 1.5 \mathrm{~g} / \mathrm{cm}^{3}\right)$. These small and slow objects, if they exist in the greater numbers that our study indicates, could account for the discrepancy between our numerical result and the observed asymmetric crater distribution. More complete observational surveys of the near-Earth asteroids can test our prediction. Future progress in the reconstruction of the true orbital distribution of NEAs by debiasing techniques would also be useful.

However, a challenge with the above explanation is that it is not easy to keep NEAs' relative velocity too low. There are many complicated resonances in the orbital zones of the terrestrial planets that can pump up the random velocities of small bodies (Michel \& Froeschlé 1997). Thus the slow population would need to be immune to these excitation mechanisms or to be continuously resupplied.

A different explanation might be that larger NEAs with very low relative velocity get fragmented due to Earth's tidal force when they approach the Earth-Moon system. This process would increase the number of projectiles (and reduce their size), and may contribute in enhancing the asymmetric distribution of craters if the fragments keep the low relative velocity of the parent body until they collide with the Moon.

A third possibility is that the lunar orbital velocity has been larger in the past. A billion years ago the lunar semimajor axis may have been as small as $\sim 90 \%$ of the current value (Bills et al. 1999), and has gradually increased to the current value due to the tidal interaction with the Earth. When the lunar semimajor axis was $90 \%$ of the current value, the lunar orbital velocity with respect to the Earth was 17\% larger than the current value. This enhancement of the lunar orbital velocity could enhance the asymmetric distribution of impacts. But the magnitude of this effect would be limited. Even if the lunar orbital velocity has been $17 \%$ larger throughout the past one billion years, it would be only as small as $1.2 \mathrm{~km} \mathrm{~s}^{-1}$, still too small compared to the average impact velocity. This larger value would still be insufficient to explain the difference of the degree of asymmetric distribution of the actual lunar craters record (apex/antapex ratio $\sim 1.65$ ) from that of our numerical result $(\sim 1.32)$ when we consider the semi-analytic estimate of the cratering rate as a function of the lunar orbital velocity (Zahnle et al. 2001; Morota \& Furumoto 2003). Similarly, Gallant et al. (2009) performed a series of numerical simulations in order to check the effect of the smaller Earth-Moon distance, and found only a tiny change in hemispherical crater ratio for Earth-Moon distance as low as $~ 90 \%$ of the current value ( $\sim 54$ Earth radii). We must note, however, that the history of the lunar orbit is thus far predominantly based on theoretical models and is not especially well constrained by observations; there may exist an exciting possibility to place an observational constraint on the lunar orbital evolution by detailed modeling of the asymmetric lunar crater record.

A fourth possibility is that the observational lunar crater data of Morota \& Furumoto (2003) is incomplete. This crater data consists only of 222 craters of diameter $D>5 \mathrm{~km}$ covering about a third of the entire lunar surface. There is certainly room for improvement of this dataset. A recent brief report of a more extensive search for lunar rayed craters as small as $0.5 \mathrm{~km}$ diameter is not conclusive (Werner \& Medvedev 2010). A potentially important source of uncertainty and confusion in interpreting the spatial patterns in the lunar rayed craters is the uncertain ages of these craters. For example, some of the larger craters are argued to be older than what they had been thought from a study of optical maturity of their ejecta (Grier et al. 2001). Similar issues are pointed out by Werner \& Medvedev (2010). Thus, it is possible that the discrepancy could be removed with a future complete and correct dataset of young lunar craters.

Finally, we should comment on the study of Gallant et al. (2009) which already reported results that are overall rather similar to ours. The first and the largest difference of our numerical model from that of Gallant et al. (2009) is that the latter simulated the lunar impacts of NEAs from a synthetic NEA sample having fixed orbital elements with distribution following Bottke et al. (2002)'s debiased NEA population (referred to as "source orbits", restricted to 16307 in the Earth-crossing region), whereas we directly integrated the orbital evolution of 
Bottke's debiased NEA population particles (in our first stage simulation) with a steady-state dynamical model. Then, using symmetric characteristics of the orbits, Gallant et al. (2009) effectively multiplied the number of source orbits four times. Next, for each of the source orbits, a disk of $10^{5}$ particles with an identical initial velocity toward the Earth-Moon system was created, and integrated until the disk particles reach the Earth. Schematic figure of Fig. 4 in Gallant et al. (2009) explains well their numerical model of the disk; the total number of particles in their numerical model is more than $1.2 \times 10^{11}$. In comparison, our model contains a smaller number of particles $\left(0.21 \times 10^{11}\right.$ clones $)$, but we have included planetary perturbations and orbital evolution in direct numerical integrations of the NEA-like particles.

Gallant et al. (2009) concluded that their numerical result of the apex/antapex asymmetry $(1.28 \pm 0.01$ when considering the ratio of craters within $30^{\circ}$ of the apex to those within $30^{\circ}$ of the antapex) is completely consistent with the value of about $1.6 \pm 0.1$ found in the available crater data of Morota \& Furumoto (2003); they attributed the difference to the large uncertainties in the crater data. As we noted above, the difference between the dynamical model and the observations is not huge, but, somewhat differently than Gallant et al. (2009), we conclude that the the results of our numerical simulation are only marginally consistent with the observed asymmetry, and we have therefore discussed at some length several explanations for the possible discrepancy.

Gallant et al. (2009) also pointed out that the impact velocity of the cratering projectiles is $\sim 20 \mathrm{~km} \mathrm{~s}^{-1}$, somewhat higher than values commonly adopted in previous studies, and that this has ramifications for proposed matches between the lunar crater size-frequency distributions and asteroidal impactors (e.g. Strom et al. 2005). Our calculations find the average impact velocity to be $22.4 \mathrm{~km} \mathrm{~s}^{-1}$, which is even slightly higher than that of Gallant et al. (2009). Not only the average impact velocity, but also the shape of the impact velocity distribution in our model is noticeably different than in Gallant et al. (2009): it is more symmetric about the mean value in our case. We attribute these differences partly to the statistical variations of the initial conditions (both studies used Bottke et al. (2002)'s debiased $a, e, I$ distribution of NEO orbits, but the particular realizations of the set of initial conditions were done independently), and partly to the evolution of the particles prior to impact on the Moon (Gallant et al. (2009)'s numerical model does not account for this orbital evolution, but our dynamical model includes this effect). Note that even though the average impact velocity as well as the shape of the impact velocity distribution in our model are different from those in Gallant et al. (2009), the resulting asymmetry in the numerical lunar cratering is quite similar to each other.

Regarding implications for origins of crater populations, certainly the higher average impact velocity of NEAs on the Moon calls for an update of such studies, but with the caveat that this higher value is derived from the currently recognized "debiased" NEA population (which may potentially be missing a significant fraction of slow NEAs near the Earth-Moon system). We also note that the Late Heavy Bombardment projectiles that Strom et al. (2005) proposed were not NEAs with the steady-state flux but main belt asteroids directly transported from main belt resonance zones to the inner solar system; such impactors would have an average impact velocity on the Moon of about $18 \mathrm{~km} \mathrm{~s}^{-1}$ (Ito \& Malhotra 2006), quite similar to the value of $17 \mathrm{~km} \mathrm{~s}^{-1}$ adopted in the Strom et al. (2005) study.

Currently several lunar missions are underway by several countries (e.g. Normile \& Bagla 2007). They will yield improved datasets to better determine the nature of the asymmetric distribution of young craters on the Moon. On the theoretical side, it would be important to improve the dynamical models by including more complete physics (such as non-gravitational forces that may be significant in the orbital evolution of small NEAs), and to improve the model estimates of observational biases in the known NEA population particularly for those with orbital parameters similar to Earth and that are difficult to observe due to their low solar elongation angles.

\section{Summary and conclusions}

We simulated numerically the spatial distribution of impacts of near-Earth objects, using a numerical model with a steady-state population of impactors based on current estimates of the debiased near-Earth objects' orbital distribution (as provided by the model of Bottke et al. 2002). We compared the results of the simulation with the observed asymmetry of the population of rayed craters on the leading/trailing hemispheres of the Moon (as measured by Morota \& Furumoto 2003). Our results and conclusions are summarized as follows.

1. Our numerical simulation yields a leading/trailing hemispherical ratio of $1.32 \pm 0.01$ for lunar impacts by near-Earth objects. This result is similar to that of Gallant et al. (2009) who obtained the value $1.28 \pm 0.01$ from a different numerical model.

2. This result of our numerical simulation is only marginally compatible with the observed ratio of $1.65 \pm 0.16$ found by Morota \& Furumoto (2003). A possible explanation for the discrepancy is that there exists a hitherto undetected population of small objects in heliocentric orbits nearly coorbiting with Earth, whose average impact velocities on the Moon are much lower than the average impact velocity of the known near-Earth object population. Other explanations are possible, including the possibility that a more comprehensive study of young lunar craters could reveal a smaller leading/trailing asymmetry and thereby remove the discrepancy with the dynamical modeling.

3. The average impact velocity of near-Earth objects on the Moon is found to be $22.4 \mathrm{~km} \mathrm{~s}^{-1}$; Fig. 3a plots the impact velocity distribution.

4. Overall, the impact angles are isotropically distributed, but there is a noticeable deficit of oblique impacts on the trailing hemisphere of the Moon.

5. The ratio of the number of collisions with the Earth and those with the Moon is found to be $20.4 \pm 0.1$.

Acknowledgements. We wish to thank Tomokatsu Morota, Fumi Yoshida, and Robert McMillan for giving us useful information and discussions. We wish to thank the reviewer Bill Bottke for many helpful comments and suggestions that led to an improved numerical model. This study is supported by the Grant-in-Aid of the Ministry of Education of Japan (18540426/2006-2008, 21540442/20092011) and the JSPS program for Asia-Africa academic platform (2009-2011). R.M. acknowledges research funding from the USA-NSF grant (AST-0806828).

\section{References}

Bills, B. G., James, T. S., \& Mengel, J. G. 1999, J. Geophys. Res., 104, 1059 Bottke, W. F., Jedicke, R., Morbidelli, A., et al. 2000, Science, 288, 2190 Bottke, W. F., Morbidelli, A., Jedicke, R., et al. 2002, Icarus, 156, 399

Danby, J. M. A. 1992, Fundamentals of Celestial Mechanics, second edition (Richmond, Virginia: Willmann-Bell Inc.)

Gallant, J., Gladman, B., \& Ćuk, M. 2009, Icarus, 202, 371

Grier, J. A., McEwen, A. S., Lucey, P. G., Milazzo, M., \& Strom, R. G. 2001, J. Geophys. Res., 106, 32847

Housen, K. R., Schmidt, R. M., \& Holsapple, K. A. 1991, Icarus, 94, 180 Ishizaki, Y., \& Furumoto, M. 1997, Planet. People, 6, 12 (text in Japanese) 
T. Ito and R. Malhotra: Asymmetric cratering on the Moon

Ito, T., \& Malhotra, R. 2006, Adv. Space Res., 38, 817

Ivanov, B. A., Neukum, G., Bottke, W. F., \& Hartmann, W. K. 2002, in Asteroids III, ed. W. F. Bottke, A. Cellino, P. Paolicchi, \& R. P. Binzel (Tucson, Arizona: The University of Arizona Press), 603

Le Feuvre, M., \& Wieczorek, M. A. 2008, Icarus, 197, 291

Levison, H. F., \& Duncan, M. J. 1994, Icarus, 108, 18

McEwen, A. S., Moore, J. M., \& Shoemaker, E. M. 1997, J. Geophys. Res., 102, 9231

Melosh, H. J. 1989, Oxford Monographs on Geology and Geophysics, Impact Cratering: A Geologic Process (New York: Oxford University Press), 11

Michel, P., \& Froeschlé, C. 1997, Icarus, 128, 230

Morota, T., \& Furumoto, M. 2003, Earth Planet. Sci. Lett., 206, 315

Normile, D., \& Bagla, P. 2007, Science, 317, 1163

Schenk, P., \& Sobieszczyk, S. 1999, BAAS, 31, 1182

Schmidt, R. M., \& Housen, K. R. 1987, Int. J. Impact Eng., 5, 543
Shoemaker, E. M. 1962, in Physics and Astronomy of the Moon (New York: Academic Press), 283

Shoemaker, E. M., Lucchitta, B. K., Wilhelms, D. E., Plescia, J. B., \& Squyres, S. W. 1982, in Satellites of Jupiter, ed. D. Morrison (Tucson: The University of Arizona Press), 435

Strom, R. G., Malhotra, R., Ito, T., Yoshida, F., \& Kring, D. A. 2005, Science, 309,1847

Werner, S. C., \& Medvedev, S. 2010, Lunar Planet. Sci. Conf., 41, 1058

Zahnle, K. J., \& Sleep, N. H. 1997, in Comets and the Origin and Evolution of Life, ed. P. J. Thomas, C. F. Chyba, \& C. P. McKay (New York: SpringerVerlag), 175

Zahnle, K. J., Dones, L., \& Levison, H. F. 1998, Icarus, 136, 202

Zahnle, K. J., Schenk, P., Sobieszczyk, S., Dones, L., \& Levison, H. F. 2001, Icarus, 153, 111 